\title{
P216 MACROSCOPIC DESCRIPTION OF FOAM FLOW THROUGH POROUS MEDIA
}

V. ZEMSKIKH

Russian Academy of Sciences, Oil and Gas Research institute, 19-16 UI. Mary Ulianovoy, 117331 Moscow, Russia

\begin{abstract}
In this paper we investigate theoretically the possibility to describe foam flow through porous media based upon the usual equations for two phase flow of immcisible fluids through porous media. We found class of solutions which can describe the main features of foam flow during gas mobility reduction (gas-blocking regime). An expression for the "gas mobility reduction factor" has been derived from the current consideration. The analysis of this expression shows that this factor is equal to (liquid mobility without foam)/(gas mobility without foam) at liquid saturation very closed to the irreducible one. A method for calculating of the gas mobility reduction factor is proposed.
\end{abstract}

Introduction. When applying foam methods in EOR (enhanced-oil-recovery), accurate prediction of the foam performance is required which is difficult without a theoretical treatment of foam flow. Analysis of the literature in this field shows that there are two main theoretical ways of describing foam flow through porous media.

The first is based on the concept of the classic Buckley-Leverett theory of two phase flow in porous media [1,2]. In the second one, macroscopic equations for foam flow are derived by considering microscopic behavior of foam [3-5]. Both ways are connected with difficulties. The first one require additional experimental data especially for given foam flow. The second one use a lot of parameters that can not be obtained directly from the experiments. These difficulties lead to a process of foam simulation which becomes very complex and hardly understandable.

The aim of this study is to propose a simple way to describe foam flow, which is based on the macroscopic behavior of the wetting and non-wetting phases and can not be derived from usual Buckley-Leverett theory.

Main Assumptions and Relations. Let us consider the gas drainage process through a sample of the porous medium pre-saturated by aqueous surfactant solution. The both phases are assumed to be incompressible, immiscible and Newtonian. In addition to that we assume capillary pressure in lamellae is equal to capillary pressure corresponding to local saturation without foam. Although gas is not a continues phase, we will apply usual Darcy's law for water flow and gas flow too.

We can write the Darcy's laws for the wetting (aqueous phase) and non-wetting (gas) phases:

$$
\begin{aligned}
& u_{w}=-\lambda_{w}(s) \cdot \nabla p_{w} \\
& u_{g}=-\lambda_{g}(s) \cdot \nabla p_{g}
\end{aligned}
$$

The following relation for pressures in wetting and non-wetting phase is satisfied:

$$
p_{s}=p_{w}+p_{c}(s)
$$

where $p_{c}(s)$ is considered as a known function of saturation.

For a one dimensional case we can write continuity equation in the following form:

$$
u_{w}+u_{g}=u 0(t)
$$

Using the relationships (3), (4), the formulas (1) and (2) can be rewritten in the form: 


$$
\begin{aligned}
& u_{w}=F_{w}(s) \cdot\left(u_{0}(t)+\lambda_{B}(s) \cdot p^{\prime} c(s) \cdot \frac{\partial s}{\partial x}\right) \\
& u_{g}=F_{g}(s) \cdot\left(u_{0}(t)-\lambda_{w}(s) \cdot p^{\prime} c(s) \cdot \frac{\partial s}{\partial x}\right)
\end{aligned}
$$

where

$$
\begin{aligned}
& F_{w}(s)=\frac{\lambda_{w}(s)}{\lambda_{w}(s)+\lambda_{8}(s)} \\
& F_{g}(s)=\frac{\lambda_{g}(s)}{\lambda_{w}(s)+\lambda_{g}(s)}
\end{aligned}
$$

and the term $p_{c}^{\prime} c(s)$ means the derivative with respect to saturation.

Note here that classic Buckley-Leverett theory considers the case where the action of the capillary forces is completely accounted for by functions $\lambda_{w}(s)$ and $\lambda_{g}(s)$, so that expressions in parenthesizes in the equations (5) and (6) are equal to $u_{0}(t)$.

We assume that these expressions are not equal $u_{0}(t)$, if foam is involved. An additional comparison to the usual case action of the capillary forces in the direction of the flow will be taken into account. The mobility functions are assumed to be unchanged.

The Equations of Steady States Theory of Foam Flow. Let us consider the flowing of "ideal" foam, when action of the capillary forces in the flow direction is at the maximum. The "ideal" foam is always stable and exists for all saturations in the porous medium. The characteristics of the "ideal" foam flow will not depend on the type of the surfactant, its concentration and so on. . The "ideal" foam can block the liquid flow completely by capillary forces. The following equations must be satisfied:

$$
u_{w}=F_{w}(s) \cdot\left(u_{0}(t)+\lambda_{s}(s) \cdot p^{\prime} c(s) \cdot \frac{\partial s}{\partial x}\right)=0
$$

and

$$
u_{g}=F_{g}(s) \cdot\left(u_{0}(t)-\lambda_{w}(s) \cdot p_{c}^{\prime}(s) \cdot \frac{\partial s}{\partial x}\right)=u_{0}(t)
$$

Equations (9) and (10) can be considered as steady state solutions of the mass conservation law for each phase

$$
\begin{aligned}
& m \cdot \frac{\partial s}{\partial t}=\frac{\partial}{\partial x} u_{w} \\
& m \cdot \frac{\partial s}{\partial t}=-\frac{\partial}{\partial x} u_{g}
\end{aligned}
$$

Taking this fact into account, we can rewrite the equations (9) and (10) as follows:

$$
\begin{gathered}
u_{0}+\lambda_{8}(s) \cdot p^{\prime} c(s) \cdot \frac{d s}{d x}=0 \\
u_{8}=u_{0}
\end{gathered}
$$

Note that the time in equations (13) and (14) can be considered as a parameter of the process"and must be defined from additional equations. Later we will see that it is defined by volume and volumetric rate of the injected gas.

The value of $u_{0}$ is positive (the positive direction is considered as the direction of the gas flow). Function $\lambda_{g}(s)$ is always positive and function $p^{\prime} c(s)$ is always negative and finite in the interval $\left(s_{i w} ; s_{i g}\right)$, where $s_{i w}$ and $s_{i g}$ are the irreducible water and gas saturations, correspondingly. Note here that function $p^{\prime} c(s)$ corresponds to drainage process. We can conclude in this case that the following condition is always satisfied: 


$$
\frac{d s}{d x}>0
$$

Condition (15) means that function $s(x)$ must be a monotonically increasing function of the coordinate.

Let us introduce the value of saturation at the inlet end of the sample $s+(t)$, which will be found later, i.e.

$$
s(0)=s+(0, t)
$$

and the dimensionless coordinate

$$
\xi=\frac{x}{L}
$$

We will consider that, for any given moment of time, the values of the injected gas volume and rate of the gas injection are known, i.e.

$$
\begin{gathered}
V_{g}=A \cdot m \cdot u 0(t) \cdot\left(s_{i g}-s+(t)\right) \\
V_{g}=V_{g}(t)
\end{gathered}
$$

where the expression $\dot{V}_{8}$ means the derivative of the gas volume injected with respect to time. The solution of the equation (13) can be written in the form:

$$
\xi(s)=-\frac{1}{u_{0} \cdot L} \cdot \int_{s}^{s t} \lambda_{B}(s) \cdot p^{\prime} c(s) \cdot d s
$$

There will be a maximum value of the function $\xi(s)$ for a given value of $\mathrm{s}_{+}$and $u_{0}$ :

$$
\xi_{\max }=\xi\left(s+(t) ; u_{0}(t) ; s_{i g}\right)
$$

Each profile (20) defines the volume of the injected gas:

$$
V_{8}=\int_{0}^{\xi_{\text {max }}}\left(s_{i g}-s\right) \cdot d \xi=-\frac{1}{u 0 \cdot L} \cdot \int_{s_{i q}}^{s_{t}}\left(s_{i g}-s\right) \cdot \lambda_{8}(s) \cdot p^{\prime} c(s) \cdot d s
$$

Using (18), we can finally write:

$$
V_{g} \cdot \dot{V}_{8}=-\frac{s \cdot m \cdot\left(s_{i}-s+\right)}{L} \cdot \int_{s_{i q}}^{s_{+}}\left(s_{i g}-s\right) \cdot \lambda_{g}(s) \cdot p^{\prime} c(s) \cdot d s
$$

The relationships (22) and (23) can be considered like equations for the values of $\xi_{\max }$ and $\mathrm{s}_{+}$. The functions $V_{8}$ and $\dot{V}_{8}$ are considered as known.

The Properties of the Solution of the Steady States Theory of Foam Flow. Let us consider some properties of the solutions. These solutions reproduce all features of the "gas blockage" process mentioned above.

During the first stage of the process approximately one pore volume of gas displaces the liquid from the sample. In this case the value of $s_{+}$, corresponding to one pore volume injected gas, is very close to the value of $s_{i w}$.

The breakthrough time, $t_{b}$, and volume of injected gas (occupied by foam) can be estimated by the following relationships:

$$
\begin{gathered}
-\frac{1}{u_{0} \cdot L} \cdot \int_{s_{i g}}^{s+((b))} \lambda_{g}(s) \cdot p^{\prime} c(s) \cdot d s=1 \\
V_{8} \cdot \dot{V}_{8}=-\frac{S \cdot m \cdot\left(s_{i g}-s+\left(t_{b}\right)\right)}{L} \cdot \int_{s_{i q}}^{s+\left(t_{b}\right)}(s i g-s) \cdot \lambda_{B}(s) \cdot p^{\prime} c(s) \cdot d s
\end{gathered}
$$


If the value of $s+(t b)$ is rather closed to irreducible water saturation, $s_{i w}$, the volume occupied by foam will be rather close to volume of the sample pore space initially occupied by mobile water. After this stage the saturation in the sample becomes practically uniform and close to irreducible water saturation. Let us estimate the mobility reduction factor at that stage of the "gas blockage". To make this estimation, we must recognize the fact that gas penetrated in the sample must be distributed according (20). Transition from one steady state profile of the saturation to another one with lower value of $\mathrm{s}_{+}$, can occur if gas will move the liquid against the capillary resistance forces though all pore space occupied by "mobile" saturations realized in the sample. We can conclude, that mobility of gas at "gas blockage" stage is equal to mobility of the liquid at average saturation in the sample, i.e.,

$$
\lambda_{s f} \cong \lambda_{w}(s a v)
$$

According to Buckley-Leverett theory, this gas mobility without foam must be equal to the value $\lambda_{g}(\operatorname{sav})$.

In this case we can obtain the following expression for the mobility reduction factor (MRF):

$$
M R F=\frac{\lambda_{w v}\left(s_{a v}\right)}{\lambda_{g}\left(s_{a v}\right)}=\frac{\mu_{8}}{\mu_{w}} \cdot \frac{k_{w r}\left(s_{a v}\right)}{k_{g r}\left(s_{a v}\right)}
$$

At the final stage of the process "gas blockage" the sample looses ability to reduce the gas mobility. At this final stage the outlet saturation, $s$.tends to inlet one, $s_{+}$. The pressure drop through the sample can not be much more than capillary pressure difference at the saturation $s_{+}$ and $s$ :

$$
\Delta p_{c}=p_{c}\left(s_{+}\right)-p_{c}\left(s_{-}\right)
$$

When $\Delta p_{c}$ becomes less then external pressure drop, the sample looses ability to reduce the gas mobility.

If the external pressure drop higher as defined by (28) at initial moment of time, we can expect that gas will flow without reduction its mobility. In this case the dependence of the gas production on the pressure drop must have a threshold character. These types of dependencies have been observed in the experiment $[6,7]$.

\section{Conclusions.}

1. The class of solutions of the two phase flow theory of immisible fluids has been found which can describe main features of foam flow through porous media in the regime called "gas blockage".

2. An expression for MRF (gas mobility reduction factor) have been derived. This expression can be easily incorporated in simulator of two phase flow.

Acknowledgments. We acknowledge J.E. Hanssen for useful discussions on his rich experimental material, T. Holt, F. Vassenden, A. Solheim and I. Akervoll for useful discussions. V.Zemskih is also grateful to Royal Norwegian Research Council for Science for scholarship that allowed this work to be carried out. 


\section{Nomenclature}

$\begin{array}{ll}\mathrm{k} & \text { absolute permeability of sample } \\ k_{g r} & \text { gas relative permeability } \\ k_{w r} & \text { water relative permeability } \\ \mathrm{L} & \text { sample length } \\ \mathrm{m} & \text { porosity of sample } \\ \mathrm{p}_{\mathrm{g}} & \text { pressure in gas phase } \\ \mathrm{p}_{\mathrm{c}}(\mathrm{s}) & \text { capillary pressure function } \\ p^{\prime}(s) & \text { derivative of capillary pressure function with respect to saturation } \\ \mathrm{p}_{\mathrm{w}} & \text { pressure in water phase } \\ A & \text { cross-section area of sample } \\ \mathrm{s} & \text { water saturation } \\ \mathrm{s}_{+} & \text {saturation at inlet end of sample } \\ \mathrm{s} . & \text { saturation at outlet end of sample } \\ \mathrm{s}_{\mathrm{av}} & \text { average saturation in sample } \\ \mathrm{s}_{\mathrm{ig}} & \text { water saturation corresponding to irreducible gas saturation } \\ \mathrm{s}_{\mathrm{iw}} & \text { irreducible water saturation } \\ \mathrm{t} & \text { time } \\ \mathrm{t}_{\mathrm{b}} & \text { breakthrough time } \\ \mathrm{u}_{0} & \text { total Darcy velocity } \\ \mathrm{u}_{\mathrm{g}} & \text { gas Darcy velocity } \\ \mathrm{u}_{\mathrm{w}} & \text { water Darcy velocity } \\ \mathrm{u}_{\mathrm{w} 0} & \text { water Darcy velocity at inlet end of sample } \\ \mathrm{V}_{\mathrm{g}} & \text { volume of injected gas } \\ \dot{V}_{8} & \text { volumetric rate for gas injection } \\ \mathrm{x}, \mathrm{y}, \mathrm{z} & \text { space coordinates }\end{array}$

\section{Greek Symbols}

$\lambda_{8} \quad$ gas mobility in presence of foam

$\lambda_{8} \quad$ gas mobility without foam

$\lambda_{w} \quad$ water mobility

$\xi \quad$ dimensionless coordinate

$\xi_{\max } \quad$ maximum length of gas penetration

$\mu_{8} \quad$ gas viscosity

$\mu_{w} \quad$ water viscosity 


\section{References}

1. Rossen W.R., Zeilinger S.C, Jianhim Shi, Lim M.T, "Mechanistic Simulation of Foam Process in porous Media", SPE paper 28940, presented at the SPE 69th Annual Technical Conference and Exhibition held in New Orleans, LA, USA, 25-28 September, pp. 493-506, 1994.

2. Khalib Z.I., Hirasaki G.J., Falls A.H., "Effects of Capillary Pressure on Coalescence and Phase Mobilities in Foam Flowing Through Porous Media", SPE Reserve. Eng., Aug., pp. 919926,1988 .

3. Kovscek A.R., Radke C.J., "Fundamentals of Foam Transport in Porous Media", in : "Foams: Fundamentals and Applications in Petroleum Industry", edited by L.L. Schramm, Adventures in Chemistry Series 242, American Chemical Society, Washington DC, 1994, pp. 115-164.

4. Chambers K.T., Radke C.J., "Capillary Phenomena in Foam Flow Through Porous Media", in : "Interfacial Phenomena in Petroleum Recovery", edited by N.R. Morrow, Surfactant Series Science, vol.36, , Marcel Dekker Inc., New York and Basel, 1991, pp. 191-256.

5. Entov V..M. "Physical and Mathematical Modeling of Foam Flow Through Porous Media", presented at 3ed Workshop on Foam Field Applications, March, Roros, Norway, 1994.

6. Hanssen J.E., Dalland M., "Gas-Blockage Foam", in : "Foams: Fundamentals and Applications in Petroleum Industry", edited by L.L. Schramm, Adventures in Chemistry Series 242, American Chemical Society, Washington DC, 1994, pp. 319-354.

7. Hanssen J.E., Haugum P., "Gas Blockage by Non-Aqueous Foams”, SPE paper 21002, presented at SPE International Symposium on Oil Field Chemistry, Anaheim, California, February 20-22, 1991. 